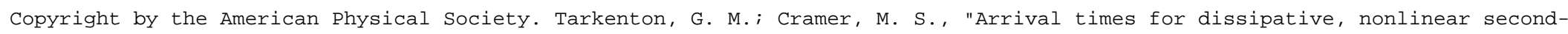
sound waves in solids," Phys. Rev. B 52, 24 DOI: http://dx.doi.org/10.1103/PhysRevB.52.24

\title{
Arrival times for dissipative, nonlinear second-sound waves in solids
}

\author{
G. M. Tarkenton \\ Institute for Advanced Physics, 8406 Red Willow Drive, Austin, Texas 78736-3004 \\ and Department of Physics, University of Texas at Austin, Austin, Texas 78712 \\ M. S. Cramer \\ Engineering Science and Mechanics Department, Virginia Polytechnic Institute and State University, \\ Blacksburg, Virginia 24061-0219 \\ (Received 17 January 1995)

\begin{abstract}
We extend our original analysis begun in Tarkenton et al. [Phys. Rev. B 49, 11794 (1994)] to include dissipative effects that are important in real cryogenic systems where nonlinear second sound exists. We present results concerning arrival times of thermal pulses propagated in cryogenic crystals, namely the behavior of the arrival times as a function of pulse amplitude. These arrival times show some surprising effects due to competing nonlinear terms: after decreasing with increasing amplitude, as one would expect, the arrival times start to lengthen due to nonlinear effects and finally saturate at a level slightly above the shortest arrival times. All these surprising effects arise from competing nonlinear terms in the expression for the wave speed. We finally relate these results to the experiment we proposed in our original paper.
\end{abstract}

In our previous work, ${ }^{1}$ we calculated the nonlinear corrections to the wave speed of a second-sound pulse in cryogenic solids. We then analyzed the structure of the resulting shock waves permitted by the theory and proposed an experiment to investigate these nonlinear effects. The experiment involved a crystal of length $H$ with a heater at one end and thermometer at the other end; the heater is switched on at time 0 and set to a fixed value of the heat flux, which sends a thermal discontinuity into the crystal. The measurement consists of timing the pulse's journey across the crystal. We showed that these arrival times should saturate for a large enough amplitude as a result of the strength-limiting (and hence speed-limiting) effects of the nonlinear terms. In that analysis, we neglected the effects of dissipation and other loss mechanisms. In Ref. 2 we outlined the effects of such phenomena on the solutions to the governing modified Burgers' equation. Here, we describe the modifications introduced by dissipation to the arrival times as a function of the initial amplitude of the pulse.

The speed of second-sound waves in cryogenic solids has the form ${ }^{1}$

$$
\lambda=U_{E 0}\left\{1+\Gamma_{0} u+\frac{1}{2} \Lambda u^{2}\right\}
$$

where $U_{E 0}$ is the linear speed, $\Gamma_{0}=o(1)$ and $\Lambda=O(1)$ are the quadratic and cubic nonlinearity parameters, respectively, and $u=\theta / \theta_{0}-1$ is the dimensionless temperature perturbation, $\theta$ being the absolute temperature and $\theta_{0}$ being the absolute temperature of the undisturbed state. (Note that in Ref. 1 what we call here $\Gamma_{0}$ was denoted $\Gamma \theta_{0}$ and $\Lambda$ was $\Lambda \theta_{0}^{2}$.)

Under the assumption that $\Lambda<0$, which is true for the materials $\mathrm{Bi}$ and $\mathrm{NaF}$, we found three different possible evolutions. $^{2}$ Case I: $\Gamma_{0}<0$; the initial discontinuity violates the admissibility condition and breaks up into a centered fan configuration. The characteristics for this case appear schematically in Fig. 1(a). If $\Gamma_{0}>0$, two different types of evolution are possible depending on the size of the initial discontinuity. Case II: if the amplitude of the initial heat pulse, $\bar{A}$, is less than $3 \Gamma_{0} / 2|\Lambda|$, then the shock simply propagates through the sample subject only to the exponential dissipative decay. The characteristics for this case appear in Fig. 1(b). Case III: if $\bar{A} \geq 3 \Gamma_{0} / 2|\Lambda|$, the initial discontinuity is inadmissible and breaks up into a centered fan-thermic shock configuration; this configuration has the complicated characteristic structure depicted in Fig. 1(c). This case differs significantly from the dissipationless case because, with no dissipation, the shock and fan remain separate entities. However, with dissipation, the shock immediately becomes nonthermic and slows down to run into the fan. This shock-fan interaction is responsible for most of the surprising physics we have found. From the characteristic structures of these cases, we can construct the arrival times for a pulse propagating in such a crystal.

In the zero-dissipation limit, we reported the arrival time data in Ref. 1 using a set of universal variables that were independent of any particular system. This follows from simple dimensional analysis, since in this case there are no natural scales in the problem. With dissipation included, the length of the crystal becomes important because dissipation introduces a natural length scale, the skin depth $L_{d}$, into the problem. (Consult Ref. 1 to find expressions for $L_{d}$ in terms of the linear wave speed and the thermal conductivity.) We can preserve the variables used in the dissipationless case by introducing a dimensionless dissipation parameter $\delta=L / L_{d}$, where $L$ is the wavelength of the wave. Then we can write the arrival 
times in terms of their deviations from the linear result

$$
\Delta=\frac{|\Lambda|}{\Gamma_{0}^{2}}\left[1-\frac{U_{E 0} t_{\mathrm{arr}}}{H}\right),
$$

where $H$ is the length of the sample and $t_{\text {arr }}$ is the arrival time; we measure the amplitude in units of $\Gamma_{0} /|\Lambda|$. For case I, the leading edge of the pulse travels with the linear speed; hence, $\Delta=0$. For the positive $\Gamma$ cases, we can represent the arrival times as follows: ${ }^{2}$

case II: $\Delta=\frac{1}{\delta \bar{H}}\left(A E_{1 H}-\frac{1}{6} A^{2} E_{2 H}\right)$;

case III: $x_{e}<H$,
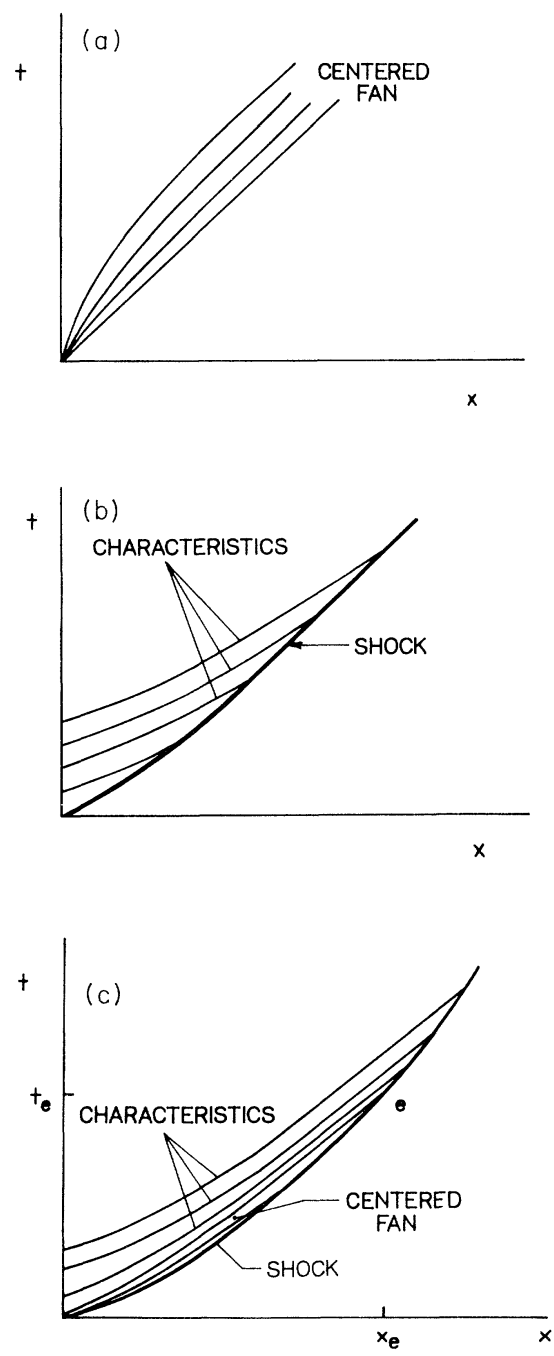

FIG. 1. Characteristic curves for the three cases outlined in the text. (a) Case I: $\Gamma_{0}<0$. The initial discontinuity violates the admissibility conditions and decays into a centered fan. (b) Case II: $\Gamma_{0}>0, A \leq 3 \Gamma_{0} / 2|\Lambda|$. The initial discontinuity propagates as a shock wave into the crystal. (c) Case III: $\Gamma_{0}>0$, $A>3 \Gamma_{0} / 2|\Lambda|$. The initial discontinuity violates the admissibility criterion and breaks up into a centered fan and thermicshock configuration. The point $e$ indicates where and when the last wave of the fan intersects the shock.

$$
\begin{aligned}
& \Delta=\frac{1}{\delta \bar{H}}\left[A\left(E_{1 H}+E_{1 e}\right)-\frac{1}{6} A^{2}\left(E_{2 H}+2 E_{2 e}\right)\right] ; \\
& \text { case III: } H<x_{e}, \Delta=\frac{1}{\delta \bar{H}}\left(2 v_{H} E_{1 H}-\frac{1}{2} v_{H}^{2} E_{2 H}\right) .
\end{aligned}
$$

Here $E_{1 H}=1-e^{-\delta \bar{H} / 2}, E_{2 H}=1-e^{-\delta \bar{H}}, x_{e}$ is the point where the last characteristic of the fan intersects the shock trajectory [see Fig. 1(c)], $E_{1 e}=1-e^{-\delta \bar{x}_{e} / 2}, E_{2 e}=1$ $-e^{-\delta \bar{x}_{e}}, v_{H}$ is a measure of the amplitude of the shock when it reaches the end of the sample (cf. Ref. 2 for specific formulas), and the overbar indicates a dimensionless quantity given by the corresponding length divided by the wavelength. These expressions have been written in order of increasing amplitude. Note that all three formulas for $\Delta$ become independent of $H$ as $\delta \rightarrow 0$, and moreover, they all become the same, indicating the scaleinvariant nature of the dissipationless case mentioned above. Plots of $\Delta$ versus $A$ appear in Fig. 2 for various values of $\delta \bar{H}=H / L_{d}$.

Figure 2 shows some interesting features. The first thing to notice is that dissipation slows the wave, since, for fixed initial amplitude, $\Delta$ is smaller for $\delta \neq 0$, as expected. The next feature is the saturation for large amplitudes; this feature occurs in the $\delta=0$ limit and persists into the dissipative case. The saturation in both cases is essentially a domain of dependence effects; the fan prevents the shock from "knowing" about any of the waves with amplitude greater than $3 \Gamma_{0} / 2|\Lambda|$. Thus the speed and, therefore, the arrival times cannot depend on the initial amplitude. Finally, in the intermediate regime, we see the wave slow down to its final, asymptotic value. For part of the pulse's journey across the crystal, the shock's speed is constant as a result of the interaction

\section{Arrival Time Deviations}

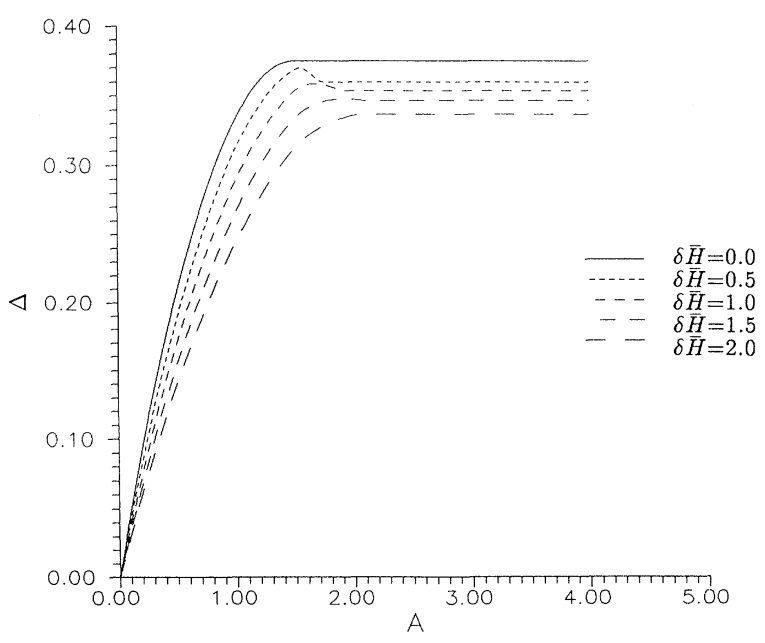

FIG. 2. Scaled arrival times $\Delta$ versus the scaled incident amplitude $A$. The initial rise comes from an increasing shock strength, while the eventual saturation is due to the shock strength being limited, and the hump for small $\delta \bar{H}$ arises from the competition between the shock-fan interaction and overall dissipation. 
with the fan. Once the shock passes $x_{e}$ (i.e., the fan disappears), it begins receiving information from the larger initial amplitudes; this causes the wave to slow, because the cubic nonlinear corrections now dominate. This causes the arrival times to increase and, hence, $\Delta$ to shrink. Thus the interplay between the nonlinear dynamics and linear dissipation creates the essential features of Fig. 2.

In our original work, we considered two specific systems: $\mathrm{NaF}$ and $\mathrm{Bi}$. Jackson and $\mathrm{Walker}^{3}$ analyze the various types of dissipation mechanisms for $\mathrm{NaF}$; they estimate that $L_{d} \approx 1.5-8.5 \mathrm{~cm}$ for $\mathrm{NaF}$ at $\theta_{0}=12 \mathrm{~K}$, depending on the impurity levels. Kopylov and MezhovDeglin $^{4}$ perform similar estimates for $\mathrm{Bi}$, finding that $L_{d} \approx 0.5 \mathrm{~cm}$ at $\theta_{0}=2.5 \mathrm{~K}$. For a crystal of length $H=1$ $\mathrm{cm}$, these values of $L_{d}$ imply that $\delta \bar{H}<1$ for $\mathrm{NaF}$, while $\delta \bar{H}>1$ for $\mathrm{Bi}$. Thus the actual behavior of the arrival times for these two materials should be somewhat different. This contrasts with the universal prediction the dissipationless analysis provides.
${ }^{1}$ G. M. Tarkenton and M. S. Cramer, Phys. Rev. B 49, 11794 (1994).

${ }^{2}$ M. S. Cramer and G. M. Tarkenton (unpublished).
${ }^{3}$ H. E. Jackson and C. T. Walker, Phys. Rev. B 3, 1428 (1971). ${ }^{4}$ V. N. Kopylov and L. P. Mezhov-Deglin, JETP Lett. 14, 21 (1971). 